\title{
Lithium exposure during pregnancy increases fetal growth
}

\author{
Eline MP Poels ${ }^{1}$ (D), Karin Sterrenburg ${ }^{2}$, André I Wierdsma ${ }^{1}$, \\ Richard Wesseloo ${ }^{1,3}$, Annemerle Beerthuizen ${ }^{1}$, Laura van Dijke ${ }^{4}$, \\ Condon Lau ${ }^{5}$, Witte JG Hoogendijk ${ }^{1}$, Hanan El Marroun ${ }^{6,7,8}$, \\ Inge L van Kamp ${ }^{4}$, Hilmar H Bijma² and Veerle Bergink ${ }^{1,9,10}$
}

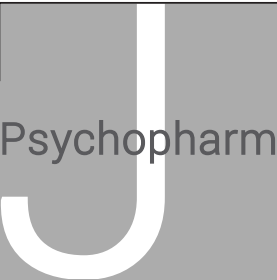

Journal of Psychopharmacology 
findings (Diav-Citrin et al., 2014; Jacobson et al., 1992; MunkOlsen et al., 2018; Newport et al., 2005). Importantly, to our knowledge no information is available relating maternal lithium therapy during pregnancy to fetal growth patterns. An environmental study showed an effect of lithium exposure through drinking water on fetal growth (Harari et al., 2015). Studying the effects of intrauterine exposure to lithium on fetal development is especially important because the intrauterine environment impacts fetal programming. Even small changes can have a large impact on the development of the child (O'Donnell and Meaney, 2017). As fetal growth is an important marker for fetal programming, the main aim of the current study is to examine the association of prenatal exposure to lithium on fetal growth and birth weight.

\section{Methods}

\section{Study design and participants}

In this retrospective observational cohort study, we collected data from two academic medical centers in the Netherlands: the Erasmus University Medical Center and the Leiden University Medical Center. Pregnant women using lithium and referred for advanced fetal ultrasound scanning between March 1994 and January 2018 were evaluated for eligibility. In the Netherlands, all pregnant women using lithium are referred for advanced 20-week ultrasound scan. All singleton pregnancies were included if there was daily lithium use from conception until 20 weeks of gestation and if data of the advanced 20 -weeks ultrasound scan were available $(n=119)$. As a control population, the Generation R Study, a prospective population-based study conducted in Rotterdam with delivery dates from 2002 until 2006, was used. All participants in the Generation R study provided written informed consent. The Generation $\mathrm{R}$ Study is designed to identify early environmental and genetic determinants of growth, development, and health from fetal life until young adulthood (Jaddoe et al., 2006). All singleton pregnancies in the Generation $\mathrm{R}$ study with information available from the start of pregnancy and data of the 20 -week ultrasound scan were included as a reference group $(n=8184)$. None of these pregnancies were lithium exposed. This study was approved by the Institutional Review Board of the Erasmus University Medical Center and conducted and reported in accordance with the STROBE guidelines.

\section{Maternal lithium use and psychiatric history}

In the lithium-exposed group, information on lithium dose, duration of use, lithium blood levels, and use of additional psychotropic medication were extracted from the medical files. Information on psychiatric diagnosis was also collected from the medical files. In the control group, information on psychotropic medication use during pregnancy was collected using two sources of information to minimize misclassification (self-reports in each trimester of pregnancy and pharmacy records) (El Marroun et al., 2012). Psychiatric symptoms were assessed by self-reported vignettes in mid-pregnancy ( $\sim 20$ weeks of gestation). Within the control group, women were categorized with a bipolar spectrum disorder if they reported at least one depression and (hypo)manic episode in history.

\section{Fetal growth}

The records of the 20-week ultrasound scans were evaluated for fetal growth measurements. The fetal growth measurements used for this study are standardized measurements and measured by well-trained and qualified sonographers in exactly the same way in both patient and control group, according to international quality standards set by the International Society of Ultrasound in Obstetrics and Gynecology (Salomon et al., 2019). In both groups, head circumference (HC), transcerebellar diameter (TCD), abdominal circumference (AC), and femur length (FL) were measured at 18-22 weeks of gestation. In both groups gestational age was assessed earlier by measuring the crown to rump length in the first trimester. TCD is generally considered to be a measure for gestational age in the second trimester of pregnancy. Estimated fetal weight was calculated by means of the Hadlock formula (Hadlock et al., 1985). Neonatal birth weight was collected from the medical files.

\section{Covariates}

Covariates were maternal age, parity, smoking, psychotropic medication use (other than lithium), body mass index (BMI) (calculated using pre-pregnancy height and weight), gestational age at birth, and child sex.

\section{Statistical analyses}

Descriptive statistics and statistical analyses were performed using the Statistical Package for the Social Sciences (version 24, IBM). The association of prenatal lithium exposure with fetal growth measurements and birth weight was analyzed using separate multiple linear regression analyses. Models for fetal growth and birth weight were adjusted for the following covariates: maternal age, maternal BMI, gestational age at the time of measurement, parity, smoking, and psychotropic medication use other than lithium (i.e., a combined covariate for the use of drugs for depression, psychosis and insomnia/anxiety). In addition, the model for birth weight was adjusted for sex of the child. Interactions between the covariates and lithium use were tested for significant improvement of the model fit. Quadratic and cubic terms for gestational age were tested for significant improvement of the model fit. Models for gestational age at birth were adjusted for maternal age, maternal BMI, parity, smoking, and psychotropic medication use other than lithium. To compare our results to previous studies (Munk-Olsen et al., 2018; Troyer et al., 1993) and assess clinical relevance, we additionally calculated odds ratios for preterm birth ( $<37$ weeks of gestation) and large for gestational age (LGA) (i.e., a birth weight above the 90th percentile of birth weight by gestational age and sex), using binary logistic regression analyses. Odds ratios were adjusted for the same covariates as described previously. All continuous covariates were centered to improve interpretation. Missing data in the covariates were handled using multiple imputations with chained equations. Missing covariate values were imputed based on birth weight, gestational age at time of ultrasound and birth, sex of the child, age of the mother, BMI of the mother, smoking status during pregnancy, psychotropic medication use during pregnancy, gravidity, and parity. A total of 10 imputed datasets were generated and analyzed. Pooled effect estimates and their $95 \%$ confidence intervals 
(CI) were reported. A two-sided $p$ value of 0.05 was considered to be statistically significant. All analyses were performed per pregnancy, not per mother. By directly comparing the lithium-exposed group to a control group from the normal population one cannot address the effect of confounding by indication (i.e., the indication for which lithium is most often described, bipolar disorder, can potentially also affect fetal growth independent of lithium exposure). To address this issue, we conducted sensitivity analyses within the control group using a selection of pregnancies in women with a broadly defined bipolar spectrum disorder but no lithium exposure and compared these with the control pregnancies in women with no bipolar spectrum disorder. Sensitivity analyses were also performed limiting the analysis to one (the first) pregnancy per mother to explore the impact of consecutive pregnancies on our results. In the lithium-exposed cohort, labor was induced as part of regular clinical practice (before 2002) around 38 weeks of pregnancy. We therefore removed pregnancies before the year 2002 in additional sensitivity analyses on gestational duration. Additional sensitivity analyses were performed after excluding pregnancies with gestational diabetes from the lithium group. The aim was to investigate whether associations of lithium with fetal growth were driven by gestational diabetes.

\section{Results}

\section{Descriptive statistics}

Descriptive characteristics are shown in Table 1. In the lithium group on average 1.4 (range 1-6) pregnancies per woman were included, versus 1.1 (range 1-3) pregnancies per woman in the control group. Maternal age and BMI were on average higher in the lithium group than the control group. Within the lithiumexposed group, most pregnancies were of women diagnosed with a bipolar spectrum disorder (i.e., bipolar I disorder, bipolar II disorder or schizoaffective disorder) $(n=110)$, other diagnoses were postpartum psychosis in history $(n=1)$ and depressive disorder $(n=8)$. Within the control group, 282 pregnancies were from women with a categorized bipolar spectrum disorder, based on self-reported lifetime experience of at least one depressive and one (hypo)manic episode. A higher percentage of women in the control group smoked throughout pregnancy (18.6\% vs $6.7 \%)$. Gestational diabetes was present in $4.2 \%$ of pregnancies in the lithium group and in $1.0 \%$ of pregnancies in the control group. Gestational age at ultrasound and sex of the child were comparable in both groups. Notably, the rate of fetal death was $2.5 \%(n=3)$ in the lithium group (one at 22 weeks due to placental insufficiency and early intrauterine growth restriction, one at 38 weeks due to a placental maturation problem, and one at 39 weeks discovered during routine check-up, possibly due to missed signs of impaired fetal condition) and $0.5 \%(n=37)$ in the control group. These fetuses were included in the fetal growth parameter analyses but not in the analyses on neonatal birth weight. The mean percentiles of $\mathrm{HC}, \mathrm{AC}$, and FL were higher in the lithium-exposed group whereas the percentile of TCD was comparable in both groups. In the lithium-exposed group the rates of premature birth and LGA were higher than in the control group.

\section{Lithium use}

Different types and various compounds of lithium were used. The most commonly prescribed was lithium carbonate, also known as
Table 1. Descriptive characteristics of the study population. ${ }^{a}$

\begin{tabular}{lcc}
\hline & $\begin{array}{c}\text { Lithium-exposed } \\
\text { group }(n=119)\end{array}$ & $\begin{array}{l}\text { Control group } \\
(n=8184)\end{array}$ \\
\hline Maternal characteristics, mean $(S D)$ & \\
Age & $34.0(4.1)$ & $29.7(5.3)$ \\
BMI & $26.3(5.8)$ & $23.6(4.4)$ \\
Bipolar spectrum disorder, & 92.4 & 3.4 \\
$\%$ & & \\
Lithium use, \% & 100 & 0.0 \\
Lithium dosage in mg, mean & 1007 & - \\
Lithium level in mmol/L, & 0.44 & -
\end{tabular}

mean

Psychotropic medication use during pregnancy other than lithium, $\%$

Drugs for depression

$16.1 \quad 1.5$

Drugs for psychosis

22.0

0.1

Drugs for anxiety/insomnia

10.1

1.5

Parity, \%

0

46.2

56.3

$>1$

53.8

43.7

Gestational diabetes, \%

4.2

1.0

Smoking habits, \%

Never smoked in pregnancy

92.4

72.7

Smoked until pregnancy was

1.0

8.7

known

Smoked throughout preg-

6.7

18.6

nancy

Child characteristics

Gestational age at ultra-

$20.2(1.2)$

$20.7(1.2)$

sound, mean (SD)

Sex of the child, \% of girls

54.3

$3(2.5)$

49.4

$37(0.5)$

$(\%)$

20-week fetal parameter percentiles, mean (SD)

Head circumference

$58.4(28.6)$

Abdominal circumference

$70.1(24.8)$

Femur length

$60.9(27.0)$

Transcerebellar diameter

$49.9(25.2)$

$48.9(28.9)$

$49.9(28.9)$

$50.3(28.9)$

$49.3(28.7)$

Birth outcome

Gestational duration in

$38.4(3.0)$

$39.8(1.9)$

weeks, mean (SD)

Premature birth, \%

15.5

5.2

Birth weight percentile,

$58.7(29.8)$

44.5 (29.6)

mean (SD)

Birth weight in grams, mean

(range)

Large for gestational age, $\%$

$3402(600-4955)$

$3412(635-5310)$

an case of missing data, valid means and percentages are presented. BMI: body mass index.

Camcolit ${ }^{\circledR}$ and Priadel ${ }^{\circledR}$. Another preparation is lithium citrate, also known as Litare ${ }^{\circledR}$. In 60 pregnancies women used lithium carbonate (Camcolit ${ }^{\circledR} n=37$, Priadel ${ }^{\circledR} n=19$, and lithium carbonate (other brands), $n=4$ ); lithium citrate was used in 38 pregnancies; in the remaining 21 pregnancies the prescription of lithium medication was unknown. The average daily lithium dosage during pregnancy was $1007 \mathrm{mg}$ divided over an average of 2.8 doses per day. Lithium citrate dosages (Litarex $564 \mathrm{mg}=6 \mathrm{mmol}$ 
Table 2. Associations of maternal lithium use in pregnancy with fetal biometric parameters at 20 weeks gestation.

\begin{tabular}{lrcr}
\hline Fetal parameter & \multicolumn{1}{c}{9} & $95 \% \mathrm{CI}$ & $p$ value \\
\hline Head circumference $(\mathrm{mm})$ & 1.77 & $0.53,3.01$ & 0.005 \\
Abdominal circumference $(\mathrm{mm})$ & 5.54 & $3.95,7.12$ & $<0.001$ \\
Femur length (mm) & 0.59 & $0.22,0.96$ & 0.002 \\
TCD (mm) & 0.13 & $-0.11,0.36$ & 0.28 \\
Estimated fetal weight (grams) & 21.05 & $12.29,29.81$ & $<0.001$ \\
\hline
\end{tabular}

Models were constructed using multiple linear regression analyses. $\beta$ represents the difference in fetal growth parameter between the lithium-exposed versus controls. Models are adjusted for: gestational age at time of ultrasound, maternal age and BMI, smoking during pregnancy, psychotropic medication use other than lithium during pregnancy, and parity.

BMI: body mass index; CI: confidence interval; TCD: transcerebellar diameter.

Table 3. Associations of maternal lithium use in pregnancy with birth weight and gestational duration.

\begin{tabular}{lrlr}
\hline Birth outcome & \multicolumn{1}{l}{ } & \multicolumn{1}{l}{$95 \%$ CI } & $p$ value \\
\hline Birth weight (grams) $^{\mathrm{a}}$ & 142.43 & $58.01,226.89$ & 0.001 \\
Gestational duration (weeks) $^{\mathrm{b}}$ & -1.41 & $-1.78,-1.05$ & $<0.001$ \\
\hline
\end{tabular}

Models were constructed using multiple linear regression analyses. $\beta$ represents the difference in birth weight in grams and pregnancy duration in weeks between the lithium-exposed versus controls.

aAdjusted for gestational age at time of birth, sex of the child, maternal age and BMI, smoking during pregnancy, psychotropic medication use other than lithium during pregnancy, and parity.

${ }^{b}$ Adjusted for maternal age and BMI, smoking during pregnancy, psychotropic medication use other than lithium during pregnancy, and parity.

BMI: body mass index; CI: confidence interval

lithium) were multiplied by 0.395 to calculate lithium carbonate dosage equivalents $(400 \mathrm{mg}=10.8 \mathrm{mmol}$ lithium). Maternal lithium plasma level during pregnancy was available for 88 pregnancies. The mean lithium level close to the time of the ultrasound, was $0.44 \mathrm{mmol} / \mathrm{L}$.

\section{Fetal growth measurements}

In Table 2 we present the associations of lithium exposure with fetal growth characteristics. Lithium use during pregnancy was significantly associated with an average increase in most growth parameters at 20 weeks of gestation, including: HC of $1.77 \mathrm{~mm}$ (95\% CI: $0.53,3.01)$, AC of $5.54 \mathrm{~mm}$ (95\% CI: 3.95, 7.12), FL of $0.59 \mathrm{~mm}(95 \% \mathrm{CI}: 0.22,0.96)$, and estimated fetal weight of 21.05 grams (95\% CI: 12.29, 29.81). Lithium use during pregnancy showed no association with TCD measurements $(\beta$ : 0.13 , 95\% CI: $-0.11,0.36)$.

\section{Birth weight and gestational duration}

The results of the multiple regression analyses on birth weight and gestational duration are presented in Table 3. Lithium use during pregnancy was significantly associated with an average increase in birth weight of 142.43 grams (95\% CI: 58.01, 226.89). In addition, lithium use during pregnancy was associated with gestational duration, with an average decrease of 1.41 weeks
(95\% CI: $-1.78,-1.05)$. Results of the binary logistic regression analyses showed that lithium use during pregnancy was associated with LGA (adjusted odds ratio $(\mathrm{OR})=1.85,95 \% \mathrm{CI}: 1.09$, 3.12 ) and premature birth (adjusted OR $=3.26,95 \% \mathrm{CI}: 1.86$, 5.74).

In the sensitivity analyses no association of bipolar spectrum disorder with 20 weeks' fetal growth, birth weight, and gestational duration was found (Table 1, Supplementary Material). The results of our analyses did not change when we limited the analyses to one pregnancy per mother, when all pregnancies before the year 2002 were removed from the analyses, and when pregnancies with gestational diabetes were removed from the lithium group.

\section{Discussion}

In this study we examined the association between lithium use during pregnancy and fetal growth parameters. In the lithiumexposed group, we found increased fetal growth at 20 weeks of gestation when compared with the control group. In addition, we found that prenatal lithium exposure was related to increased birth weight, whereas the average gestational age at birth was lower. Furthermore, lithium use during pregnancy was associated with LGA birth weight and preterm birth. No association of maternal bipolar spectrum disorder and fetal growth was found, which may potentially suggest a specific effect of lithium exposure during pregnancy on fetal growth.

An environmental study by Harari et al. found a non-significant inverse association between lithium exposure during pregnancy and fetal size in the second trimester in a study of 194 pregnancies in the Argentinean Andes (Harari et al., 2015). In addition, they found a significant association between lithium exposure during pregnancy and birth length but no association with birth weight. In this study, lithium exposure was through drinking water in relatively very low concentrations (median $25 \mu \mathrm{g} / \mathrm{L}$, which corresponds to $0.0036 \mathrm{mmol} / \mathrm{L}$, i.e., 122 times lower than the mean lithium blood concentration in our study), which could explain the difference in our findings. It is possible the increases in fetal growth and birth weight found in our study are not present when lithium blood concentration is low. The association of lithium and higher neonatal birth weight was previously found in a prospective cohort study by Jacobson et al. (Jacobson et al., 1992). Three other studies reported no differences in birth weight between lithium exposed and non-exposed neonates (Diav-Citrin et al., 2014; Munk-Olsen et al., 2018; Troyer et al., 1993). Importantly, the largest study on this subject investigated only the association of lithium with low birth weight and being small for gestational age $(<10$ th percentile) (MunkOlsen et al., 2018). In our study, we found an association of lithium use during pregnancy with an average increase in birth weight of 142 grams and with LGA. The magnitude of our findings strengthens the clinical relevance of our results. There are several effects of a high birth weight on the child, both short and long term. Short-term effects are shoulder dystocia (obstructed delivery of the shoulder due to mechanical issues), perinatal asphyxia, hypoglycemia, and longer hospital stay (Junior et al., 2017). Long-term effects are increased risk of obesity from childhood to early adulthood, as well as an increased risk of cardiovascular disease (Yu et al., 2011). 
An increased risk of preterm birth was previously reported in two studies including women who use lithium during pregnancy (Diav-Citrin et al., 2014; Troyer et al., 1993). Our results are consistent with these studies, although the mechanism of this association remains unclear. Our sensitivity analysis on the gestational duration association showed that results remained unchanged after excluding all pregnancies before 2002 (in which labor was induced as part regular clinical practice) from the analysis. This is particularly important because data on the lithium group were collected over a long period of time (between 1994 and 2018) and due to a change in clinical hospital guidelines in this time period, the induction of labor around 38 weeks pregnancy became less common after 2002. The association of lithium with pregnancy duration should be interpreted very carefully, as residual confounding by indication is possible as symptom severity of bipolar disorder or side effects from lithium could have influenced healthcare professionals to induce labor prematurely, also after 2002.

Further, our results suggest that lithium use during pregnancy is associated with increased fetal growth, already measurable at around 20 weeks of gestational age. The mechanism of this association should be investigated further, for example in animal models. As an interesting hypothesis in this context we propose the role of brain-derived neurotrophic factor (BDNF). Recent research has indicated that BDNF plays a crucial role in fetal metabolic programming through regulation of energy homeostasis and by regulating glucose metabolism in peripheral tissues (Briana and Malamitsi-Puchner, 2018). Further, it has been shown that placental BDNF gene expression is upregulated in maternal type 1 diabetes and gestational diabetes mellitus, and downregulated in neonates with non-diabetic macrosomia, compared with normal birth weight neonates (Briana and MalamitsiPuchner, 2018). Interestingly, lithium use has been associated with increased BDNF levels (Malhi et al., 2013; Tunca et al., 2014). Hence, one of the possible mechanisms underpinning the association between lithium use and increased fetal growth might be that lithium results in increased maternal serum BDNF, which might subsequently result in altered regulation of placental BDNF. This in turn could alter the regulation of fetal growth by interfering with the energy homeostasis and regulating glucose metabolism. In this scenario, the effect of maternal lithium use on fetal growth most likely reflects altered fetal programming with possible long-term impacts.

Thanks to a collaboration between Erasmus University Medical Center and Leiden University Medical Center, we were able to include a large number of women with bipolar disorder who used lithium during pregnancy. Also, thanks to a collaboration with the Generation R study, we were able to compare this group with a representative sample of the general population, which increased the power of this study. The results of this study need to be interpreted with a few limitations in mind. As in any observational study, we cannot rule out residual confounding, namely, unmeasured factors associated with both lithium and fetal growth. For example, we did not have detailed information on education and socioeconomic status in the lithium group and were therefore not able to adjust for these possible confounders. However, it is unlikely that our results are driven by a confounding effect of socioeconomic status. Bipolar disorder is associated with lower socioeconomic status (Schoeyen et al., 2011) and low socioeconomic status has been associated with lower birth weight
(Jansen et al., 2009). On the contrary, we find fetal growth to be increased in the lithium group. Other possible confounding factors of importance might be related to the severity of the psychiatric disease, as well as dosing regimens and serum levels of lithium, glucose metabolism, and nutrition. Also, our study was not designed to study trimester specific effects since we assessed fetal growth at one time point during pregnancy. We did have birth weight data, enabling us to conclude that the effect we found at around 20 weeks of gestation was still present at birth. Data from the lithium-exposed group were collected over a longer time period (between 1994 and 2018) whereas data from the control group were collected between 2002 and 2006. Ideally data would have been collected in the same time period although we believe our outcomes of interest (i.e., fetal growth and birth weight) are relatively stable over time. Lastly, we cannot rule out an effect of confounding by indication, even though we did not find an association between fetal growth and unmedicated bipolar spectrum disorder in our control group. In our study, we investigated the influence of bipolar disorder without medication on fetal growth, by running sensitivity analyses on women with self-reported bipolar spectrum disorder $(n=282)$. However, these women likely have a less severe form of bipolar spectrum disorder compared with the lithium-exposed patients because diagnosis is based on self-reported vignettes. Because detailed information on psychiatric history was not available for a large part of the population, sensitivity analysis correcting for illness severity was unfortunately not possible.

Our results are important for the estimation of all effects associated with lithium use during pregnancy. These effects may not only be limited to pregnancy but might also be carried into child development later in life. The theory of Developmental Origins of Health and Disease refers to the concept that early life events could explain an individual's risk for non-communicable disease in later life (Barker, 1990). According to this theory, the intrauterine environment can change fetal programming, which bears an impact on much more than just birth weight, including changes in physiology and metabolism. This means small prenatal changes, such as the increased fetal growth we found in our study, can have impact in later life (Hanson and Gluckman, 2014). As an example, LGA fetuses have an increased risk of developing components of the metabolic syndrome during childhood (Boney et al., 2005). Only one study investigated the postnatal growth of 15 children exposed to lithium in utero and reported outcomes within the normal range (van der Lugt et al., 2012). Therefore, there is an urgent need for studies with a larger sample size. The rate of fetal death, also before 20 weeks of gestational age, clearly warrants further investigation.

Women of a fertile age who use lithium should be preconceptionally informed about the potential risks and benefits associated with lithium use during pregnancy. Together with their treating physician, women should weigh up the benefits of lithium treatment during pregnancy against the risks of adverse fetal and neonatal (long-term) outcomes and compare these risks with those associated with the use of alternative medication.

\section{Conclusion}

This study investigated the effect of lithium use during pregnancy on fetal growth as an indicator of fetal programming. We found associations of prenatal lithium exposure and increased 
fetal growth, which may have health implications in later life. Knowledge of unintended consequences of maternal lithium use during pregnancy is important for establishing clinical guidelines to optimize the treatment of bipolar disorder during the perinatal period.

\section{Declaration of conflicting interests}

The author(s) declared no potential conflicts of interest with respect to the research, authorship, and/or publication of this article.

\section{Funding}

The author(s) received no financial support for the research, authorship, and/or publication of this article.

\section{Ethics statement}

The study was approved by the medical ethical review board of Erasmus University Medical Center (MEC-2017-1177).

\section{ORCID iD}

Eline MP Poels (iD https://orcid.org/0000-0003-2134-3929

\section{Data availability}

KS, LvD, EMPP, HM, RW, and VB had access to the study data. Access to the data is ongoing for EMPP and VB.

\section{Supplemental material}

Supplemental material for this article is available online.

\section{References}

Barker DJP (1990) The fetal and infant origins of adult disease. Br Med J 301: 1111.

Boney CM, Verma A, Tucker R, et al. (2005) Metabolic syndrome in childhood: Association with birth weight, maternal obesity, and gestational diabetes mellitus. Pediatrics 115: e290-e296.

Briana DD and Malamitsi-Puchner A (2018) Developmental origins of adult health and disease: The metabolic role of BDNF from early life to adulthood. Metabolism 81: 45-51.

Diav-Citrin O, Shechtman S, Tahover E, et al. (2014) Pregnancy outcome following in utero exposure to lithium: A prospective, comparative, observational study. Am J Psychiatry 171: 785-794.

El Marroun H, Jaddoe VW, Hudziak JJ, et al. (2012) Maternal use of selective serotonin reuptake inhibitors, fetal growth, and risk of adverse birth outcomes. Arch Gen Psychiatry 69: 706-714.

Fornaro M, Maritan E, Ferranti R, et al. (2020) Lithium exposure during pregnancy and the postpartum period: A systematic review and meta-analysis of safety and efficacy outcomes. Am J Psychiatry 177: 76-92.

Geddes JR and Miklowitz DJ (2013) Treatment of bipolar disorder. Lancet 381: 1672-1682.

Hadlock FP, Harrist RB, Sharman RS, et al. (1985) Estimation of fetal weight with the use of head, body, and femur measurements-A prospective study. Am J Obstet Gynecol 151: 333-337.

Hanson MA and Gluckman PD (2014) Early developmental conditioning of later health and disease: Physiology or pathophysiology? Physiol Rev 94: 1027-1076.
Harari F, Langeen M, Casimiro E, et al. (2015) Environmental exposure to lithium during pregnancy and fetal size: A longitudinal study in the Argentinean Andes. Environ Int 77: 48-54.

Jacobson SJ, Jones K, Johnson K, et al. (1992) Prospective multicenter study of pregnancy outcome after lithium exposure during 1st trimester. Lancet 339: 530-533.

Jaddoe VW, Mackenbach JP, Moll HA, et al. (2006) The Generation R Study: Design and cohort profile. Eur J Epidemiol 21: 475-484.

Jansen PW, Tiemeier H, Looman CW, et al. (2009) Explaining educational inequalities in birthweight: The Generation R Study. Paediatr Perinat Epidemiol 23: 216-228.

Junior EA, Peixoto AB, Zamarian AC, et al. (2017) Macrosomia. Best Pract Res Clin Obstet Gynaecol 38: 83-96.

Malhi GS, Tanious M, Das P, et al. (2013) Potential mechanisms of action of lithium in bipolar disorder. Current understanding. CNS Drugs 27: 135-153.

Merikangas KR, Jin R, He JP, et al. (2011) Prevalence and correlates of bipolar spectrum disorder in the world mental health survey initiative. Arch Gen Psychiatry 68: 241-251.

Munk-Olsen T, Liu X, Viktorin A, et al. (2018) Maternal and infant outcomes associated with lithium use in pregnancy: An international collaborative meta-analysis of six cohort studies. Lancet Psychiatry 5: 644-652.

Newport DJ, Viguera AC, Beach AJ, et al. (2005) Lithium placental passage and obstetrical outcome: Implications for clinical management during late pregnancy. Am J Psychiatry 162: 2162-2170.

Nora JJ, Nora AH and Toews WH (1974) Letter: Lithium, Ebstein's anomaly, and other congenital heart defects. Lancet 2: 594-595.

O'Donnell KJ and Meaney MJ (2017) Fetal origins of mental health: The developmental origins of health and disease hypothesis. Am J Psychiatry 174: 319-328.

Patorno E, Huybrechts KF and Hernandez-Diaz S (2017) Lithium use in pregnancy and the risk of cardiac malformations. $N$ Engl J Med 377: 893-894.

Salomon LJ, Alfirevic Z, Da Silva Costa F, et al. (2019) ISUOG practice guidelines: Ultrasound assessment of fetal biometry and growth. Ultrasound Obstet Gynecol 53: 715-723.

Schoeyen HK, Birkenaes AB, Vaaler AE, et al. (2011) Bipolar disorder patients have similar levels of education but lower socio-economic status than the general population. J Affect Disord 129: 68-74.

Tondo L, Alda M, Bauer M, et al. (2019) Clinical use of lithium salts: Guide for users and prescribers. Int J Bipolar Disord 7: 16.

Troyer WA, Pereira GR, Lannon RA, et al. (1993) Association of maternal lithium exposure and premature delivery. J Perinatol 13: 123-127.

Tunca Z, Ozerdem A, Ceylan D, et al. (2014) Alterations in BDNF (brain derived neurotrophic factor) and GDNF (glial cell line-derived neurotrophic factor) serum levels in bipolar disorder: The role of lithium. J Affect Disord 166: 193-200.

van der Lugt NM, van de Maat JS, van Kamp IL, et al. (2012) Fetal, neonatal and developmental outcomes of lithium-exposed pregnancies. Early Hum Dev 88: 375-378.

Viguera AC, Whitfield T, Baldessarini RJ, et al. (2007) Risk of recurrence in women with bipolar disorder during pregnancy: Prospective study of mood stabilizer discontinuation. Am J Psychiatry 164: 1817-1824; quiz 1923.

Wesseloo R, Kamperman AM, Munk-Olsen T, et al. (2016) Risk of postpartum relapse in bipolar disorder and postpartum psychosis: A systematic review and meta-analysis. Am J Psychiatry 173: 117-127.

Yu ZB, Han SP, Zhu GZ, et al. (2011) Birth weight and subsequent risk of obesity: A systematic review and meta-analysis. Obes Rev 12: $525-542$. 